\title{
Correlation of CT-scan findings of lung lesions and pathologic diagnosis
}

\begin{abstract}
Background: we aimed to evaluate the features of lung nodules and masses in $\mathrm{Ct}$ scan that help to distinguish malignant lesions from benign ones.

Method: The study was performed on 50 pulmonary lesions biopsied at the Shahid Faghih Hospital by an interventional radiologist. CT scan findings were evaluated by a radiologist and compared with pathologic outcomes. Data was entered into SPSS software and by Descriptive statistical methods of frequency and Roc curve analysis and Chi-squared test and T-test at the level of alpha 0.05 was analyzed.

Results: The relationship between smoking and malignancy was determined in this study. There was no significance found in mean age and sex, lesion density and enhancement of benign and malignant nodules. Smooth border was mostly seen in benign lesions and lobulated and spiculated borders in malignant lesions. Popcorn calcification was in favor of benignity and calcification in periphery of lesion was in favor of malignancy. Cavity was mostly seen in benign lesions

Conclusion: smoking and the spiculated or lobulated margins, calcification in the periphery of the lesion were associated with malignant lesions, and the smooth margin and popcorn calcification and cavity formation were mostly seen in benign lesions. Enhancement showed no significance.
\end{abstract}

Keywords: CT scan, pulmonary nodule, enhancement, density, border, calcification
Volume 6 Issue 3 - 2019

\section{Seyed Hamed Jafari, Mohammad Reza Sasani, Mohammad Javad Athari,Amin Dehdashtian, Fereshte Bagheri}

Department of Radiology, Medical Imaging Research Center, Shiraz University of Medical Sciences, Iran

Correspondence: Fereshte Bagheri, Department of Radiology, Nemazee Hospital, Shiraz University of medical sciences, Shiraz, Iran,Tel 0098-9|76046722, Email fereshteebagherii@gmail.com

Received: July 28, 2019 | Published: August 15, 2019
Abbreviations: SPN, solitary pulmonary nodule; SD, standard deviation

\section{Introduction}

Lung cancer is today the most common cause of deaths by cancer in the world. Therefore, it accounts for a huge burden on public health system. ${ }^{1,2}$ Previous studies have revealed if it is diagnosed early enough, $75 \%$ response to treatment could be obtained since it is easier to cure in early stages with fewer complications. ${ }^{3}$

A solitary pulmonary nodule (SPN) is an approximately round lesion completely surrounded by pulmonary parenchyma with the size of less than $3 \mathrm{~cm}$ in diameter. The incidence of cancer in patients with solitary nodules ranges from 10 to 70 percent. Of the benign causes, infectious granulomas are responsible for about 80 percent, and hamartomas about 10 percent. $^{4}$

Solitary pulmonary nodules (SPNs) have always been a hot issue in radiological image research in order to distinguish benign from malignant nodules without surgery since the diagnosis of benignity or malignancy of SPNs is completely related to the future treatment planning.

CT scan as one of the most frequent modalities for lung cancer raises the question of what the best approach is toward the identified SPNs. ${ }^{5}$

In this study we aimed to evaluate some of radiologic characteristics of SPN and assess morphologic features that can help differentiate benign and malignant lesions.

\section{Method}

We retrospectively reviewed SPNs detected on screening or incidentally found, then undergone Tru-cut biopsy under guide of CT scan and pathologically confirmed at Faghihi Hospital (Shiraz, Iran) between March 2016 and April 2017. Fifty patients with SPNs that were found incidentally were included if there was no history of a chest trauma or respiratory infection that could explain their presence. During the mentioned period of time, 88 patients were biopsied in this Center. 29 were excluded due to lack of chest CT imaging and 9 due to inaccessibility to pathology results. The Participants were between 24 and 85 years of age.

All the collected imagings of patients were done in Nemazee and Faghihi hospitals (Shiraz, Iran) by a16 slice spiral CT (GE medical systems, Milwaukee, WI, USA) and a routine scanning $120 \mathrm{Kvp}$ and slice thickness of $1.25-2 \mathrm{~mm}$, followed by dynamic enhanced scanning. $1.20-5 \mathrm{cc} / \mathrm{kg}$ (maximum: $100 \mathrm{cc})$ of Visipaque $(320 \mathrm{mgI} / \mathrm{ml})$ was injected for the scan through the cubital vein with a flow rate of $2.5-3 \mathrm{ml} / \mathrm{s}$. contrast enhanced scanning of the lung was performed after 30 seconds.

All CT images were read by an experienced radiologist and interpreted both at lung window and mediastinal settings.

These nodules were evaluated based on morphology and margin characteristics, size, calcification, enhancement, and cavity formation. Nodule size was determined by measuring the maximal diameter. Nodule margins were labeled as lobulated when a portion of the nodule's surface demonstrated a wavy or scalloped shape and as spiculated when strands extended from the nodule margin into the lung parenchyma.

The experimental protocol was approved by Ethics Committee of Shiraz University of medical sciences by the number of IR.sums.med. rec.1396.s280

Retrospectively, the entire biopsied tissue specimens were fixed in formalin and embedded in paraffin. Several $10 \mu \mathrm{m}$-thick sections 
were taken and stained with hematoxylin-eosin and examined using light microscopy.

The acquired data are expressed as mean \pm standard deviation (SD). For comparison purposes, patients were divided into two groups, according to their final diagnosis, in malignant and non-malignant etiologies of the solitary pulmonary cavities. The variables of the groups were analyzed using independent-sample Student's t-test, when numerical, and using the Chi-square test when categorical. All tests were done via SPSS ${ }^{\circledR}$ software (18.0, IBM ${ }^{\circledR}$, USA) program and $\mathrm{P}$-value $\leq 0.05$ considered as statistically significant.

\section{Results}

The images of subjects of this study in which 29 were males and 21 were female, with a mean age of 55.3 \pm 17.70 , were reviewed. Among them, 8 had positive history of malignancy ( 2 of them pulmonary, and 6 extra pulmonary involvements).

26 lesions were diagnosed as non-malignant and 24 as malignant. Among the nonmalignant diagnoses, 5(19\%) and among malignant ones $18(75 \%)$ had a history of smoking which showed statistically significant difference between the two group $(p<0.001) .78 \%$ of smoker participants had malignant lesion.

There were no noticeable difference seen between sex and the etiology of SPNs (malignant: 14male and 10 female vs. benign: 15 male and 11 female; $\mathrm{P}=1)$.

The average age of patients with malignant lesion was 58.4 compared to 53.6 in non-malignant group which showed no significant difference $(\mathrm{P}>0.33)$.

In the current study, based on ROC analysis, a nodule with size of more than $19 \mathrm{~mm}$ had the highest sensitivity (91.70) and specificity (46.20) to predict malignancy with $\mathrm{AUC}=0.67$ and $\mathrm{p}$ value $<0.05$.

No significant discrepancy was noted in density of nodule in nonenhanced images in the two groups (max: 61, 65; min: 7, -102, mean: 34.6, 25.10; in malignant and non-malignant group, respectively).

Based on ROC analysis to differentiate a malignant lesion, a nodule with contrast enhancement of more than $3 \mathrm{HF}$ had the highest sensitivity $(100 \%)$ and specificity $(34.60 \%)$ with accuracy $=10 \%$. Considering low accuracy, enhancement of nodule more than $3 \mathrm{HF}$ is not a reliable factor to discriminate benign from malignant lesions. It is noteworthy that previous data considered $15 \mathrm{HF}$ as a cutoff point which showed sensitivity $=58.30 \%$, specificity $=69.20 \%$, and accuracy $=64 \%$ in this study.

$95 \%$ of lesions with regular margin were benign. $81.30 \%$ of speculated border lesions and $71.40 \%$ of lobulated ones were malignant.

In this study, 6 lesions showed calcification which 2 of them had popcorn appearance with benign pathology result and 6 showed foci of tiny calcification with malignant etiology. 6 cavitary lesions were found with 4 benign origin and 2 malignant.

\section{Discussion and conclusion}

The management algorithm of pulmonary nodules (found at baseline scans) is mostly based on size and the consistency of the nodules (solid, partial-solid or non-solid). ${ }^{6}$ We observed in our study that the age, nodule density and enhancement tended to have a lower value in the benign nodules, without reaching statistical significance. It was also noticed that malignant nodules are larger and more often non-spherical, irregular, lobulated or spiculated than benign lesions. Popcorn calcification and cavitary lesions were also noted in benign lesions despite foci of tiny calcifications seen in malignant ones.

Previous studies demonstrated that nodule size was a good marker of the probability of malignancy. The possibility of malignancy progressively raised with increasing nodule size. Haiyang et al. showed that the rate of malignancy in nodules with diameter $\leq 10 \mathrm{~mm}$ was $56 \%$ in comparison to $88.70 \%$ in nodules $>10 \mathrm{~mm}$. another study with the consistent result showed that $47.40 \%$ of pulmonary nodules measuring $<10 \mathrm{~mm}$ were malignant, while $70.10 \%$ of nodules measuring $>10 \mathrm{~mm}$ were malignant. This proposes a close connection between nodule size and malignancy. ${ }^{7}$ Similarly, based on the result of current study, larger diameters had more correlation with malignant etiologies and a nodule with size of more than $19 \mathrm{~mm}$ had the highest sensitivity and specificity to predict malignancy.

In a study by Xua et al., baseline nodule density cannot be used to distinguish benign from malignant pulmonary nodules, but an increase in density is indicative of malignancy and needs a shorter follow-up or a biopsy. ${ }^{9}$ Another study showed that the threshold of $20 \mathrm{HU}$ for the net enhancement had high sensitivity, accuracy, positive predicative value and negative predicative value for diagnosing benign and malignant nodules are relatively high. ${ }^{10}$ This threshold was determined $3 \mathrm{HU}$ in our study which had low accuracy. So despite previous studies, acquired results of the current study revealed that enhancement is not a proper predictive factor for evaluation of malignancy in a pulmonary nodule.

Haiyang et al. also determined that the presence of lobulation or an air cavity density were predictive of malignancy, while the absence of spiculation and pleural tag were associated with benignity which is partly competent with the results of our study. ${ }^{8}$

Some evidence manifested that specific patterns of calcification within a SPN (diffuse, central, laminated or concentric, or popcorn) are specific for a benign lesion However, no specific pattern is known for malignancy. ${ }^{11}$ In the present study, popcorn calcification was seen in benign lesions and tiny foci of calcification in malignant ones however not statistically significant.

Previous investigation showed that cavity wall thickness $<5 \mathrm{~mm}$ leads to a benign etiology, whereas irregular and thicker walls more than $15 \mathrm{~mm}$ are more prevalent in malignant lesions, although this is not always the rule. ${ }^{12}$ In our study, 6 cavitary lesions were found with 4 benign origin and 2 malignant. One of them had wall thickness more than $15 \mathrm{~mm}(21 \mathrm{~mm})$ which was malignant. Among those with wall thickness less than $15 \mathrm{~mm}, 4$ were benign and 1 was malignant.

To sum up, chest CT imaging is a valuable modality for the characterization of solitary lung nodules in order to treatment planning and avoids unnecessary actions. There are several limitations to the current study, which means that further evaluation is essential. Our study is limited by the relatively small numbers of cases. Also, the evaluation of lung nodules was subjective, which may lead to selection bias. It is worth mentioning that this was a retrospective research on nodules undergone Tru-cut biopsy to discriminate malignant from benign lesions so pre-test risk factor evaluation was not possible. 


\section{Funding}

None.

\section{Acknowledgements}

This study was from the thesis of the degree of specialty in radiology (Project no. 96-01-01-14066 ) of Mohammad Javad Athari and supported by Shiraz University of medical sciences, Shiraz, Iran.

\section{Conflict of interest}

The authors declare that there is no conflict of interest.

\section{References}

1. Manser R, Irving L, Byrnes G, et al. Screening for lung cancer: a systematic review and meta-analysis of controlled trials. Thorax. 2003;58(9):784-7899.

2. Pasic A PP, Sutedja TG. What is early lung cancer? A review of the literature. Lung Cancer. 2004;45(3):267-77.

3. Mahersia H, Zaroug M, Gabralla L. Lung cancer detection on CT scan images: a review on the analysis techniques. IJARAI. 2015;4(4):38-45.

4. Siegelman SS, Khouri N, Leo F, et al. Solitary pulmonary nodules: CT assessment. Radiology. 1986;160(2):307-312.
5. Ost D, Fein AM, Feinsilver SH. The solitary pulmonary nodule. New England Journal of Medicine. 2003;348(25):2535-2542.

6. MacMahon H, Naidich DP, Goo JM, et al. Guidelines for management of incidental pulmonary nodules detected on CT images: from the Fleischner Society 2017. Radiology. 2017;284(1):228-243.

7. Shi C-Z, Zhao Q, Luo LP, et al. Size of solitary pulmonary nodule was the risk factor of malignancy. $J$ Thorac Dis. 2014;6(6):668-676.

8. $\mathrm{Hu} \mathrm{H}$, Wang Q, Tang $\mathrm{H}$, et al. Multi $\square$ slice computed tomography characteristics of solitary pulmonary ground $\square$ glass nodules: Differences between malignant and benign. Thoracic cancer. 2016;7(1):80-87.

9. Xu DM, van Klaveren RJ, de Bock GH, et al. Role of baseline nodule density and changes in density and nodule features in the discrimination between benign and malignant solid indeterminate pulmonary nodules. Eur J Radiol. 2009;70(3):492-498.

10. Jiang N-C, Han P, Zhou C-K, et al. Dynamic enhancement patterns of solitary pulmonary nodules at multi-detector row CT and correlation with vascular endothelial growth factor and microvessel density. $A i$ Zheng. 2009;28(2):164-169.

11. Yankelevitz DF, Henschke CI. Derivation for relating calcification and size in small pulmonary nodules. Clin Imaging. 1998;22(1):1-6.

12. Woodring JH, Fried AM. Significance of wall thickness in solitary cavities of the lung: a follow-up study. AJR Am $J$ Roentgenol. $1983 ; 140(3): 473-474$. 